\title{
Die Endoprothetik des oberen Sprunggelenkes - derzeitiger Stand
}

\author{
Sebastian Lieske, Katja Schenk, Michael John, Hans Wolfram Neumann
}

\section{Zusammenfassung}

In den letzten Jahren zeigen sich zunehmend bessere Mittel- und Langzeitergebnisse nach Endoprothesenimplantation am Sprunggelenk. Sowohl die verbesserten Implantate und Oberflächenbeschichtungen als auch die wachsende Erfahrung der Operateure haben einen großen Anteil an diesem Erfolg. In der Orthopädischen Universitätsklinik Magdeburg wurden zwischen 1996 und 2006 570 Sprunggelenksendoprothesen implantiert. In 178 Fällen (31,2\%) bestand eine primäre, in 73 Fällen $(12,8 \%)$ eine Arthrose bei rheumatoider Arthritis und in 319 Fällen (56\%) eine posttraumatische Arthrose. Subjektiv waren $82 \%$ der Patienten mit dem postoperativen Ergebnis zufrieden bzw. sehr zufrieden und $18 \%$ mäßig bzw. nicht zufrieden. Der Range of motion (ROM) verbesserte sich von präoperativ $27^{\circ}$ auf postoperativ $33^{\circ}$. Neben der präoperativen Planung und dem Erfahrungslevel des Operateurs („learning curve“), ist das postoperative Management (inkl. Physiotherapie) von entscheidender Bedeutung für den Erfolg der Behandlung.

\section{Endoprosthetics for the Ankle Joint - Current Status}

Recently, an increasing number of good middle- and long-term results have been reported after implantations of ankle joint prosthetics. Not only improved implants and surface treatments but also the increasing experience of surgeons has made a major contribution to this success. In the Department of Orthopaedics of Magdeburg University between 1996 and 2006570 ankle joint replacements were carried out. In 178 cases (31.2\%) there was primary arthrosis, in 73 cases $(12.8 \%)$ arthrosis with rheumatoid arthritis, and in 319 (56\%) cases post-traumatic arthrosis. Subjectively, $82 \%$ of the patients were satisfied or very satisfied with the postoperative results whereas $18 \%$ were only moderately or not satisfied. The range of motion (ROM) improved from preoperatively $27.0^{\circ}$ to $33.0^{\circ}$ postoperatively. Besides preoperative planning and the learning curve of the surgeon, the postoperative management (including physiotherapy) is of decisive importance for the success of the treatment.

\section{Einleitung}

Vor einigen Jahren war die Sprunggelenksendoprothetik ein Verfahren, das nur wenige Protagonisten hatte. Obwohl bereits 1973 die erstmalige Implantation einer Endoprothese durch G. Lord und J. H. Marotte erfolgte, und somit schon über 30 Jahre Innovation und Entwicklung hinter uns lagen, konnte sie sich zunächst nur schwerlich

OP-JOURNAL 2007; 23: 98 - 103

(c) Georg Thieme Verlag KG Stuttgart • New York behaupten [12]. Sie blieb lange Zeit ein Eingriff für spezialisierte Zentren. In unserer Einrichtung haben wir 1990 die ersten Erfahrungen mit Sprunggelenksprothesen gesammelt. Folgende Kriterien für eine Implantation waren damals für uns ausschlaggebend: Zum einen gab es die Patientengruppe, bei denen die Gelenkdestruktion soweit fortgeschritten war, dass - bis auf eine Arthrodese - keine weiteren Therapieoptionen vorhanden waren. Zum anderen gab es die Patienten die bei geringen radiologischen Veränderungen über massive Beschwerden klagten. Hier war also die
Operation aufgrund der gut erhaltenen Gelenkanatomie auf den ersten Blick einfacher. Leider hatten wir mit den ersten 5 Fällen Patienten mit schwer destruierten Gelenken gewählt. Die „Niederlage“ war hier vorprogrammiert. Wir implantierten diesen Patienten 1990 eine Buechel-Pappas-Prothese. Innerhalb kurzer Zeit wurden 4 revidiert, die Endoprothese entfernt und eine Arthrodese durchgeführt. Lediglich bei einem Patienten hatten wir eine Überlebenszeit der Prothese von 15 Jahren, allerdings mit einer Revision im Verlauf. Daraufhin führten wir von 1991-1996 nur noch Arthrodesen durch.

Bis dato gilt vielerorts die Arthrodese als der Goldstandard ohne Alternativen. Sie führt bei verschiedenen Techniken zu guten Ergebnissen [7,13]. Einige Autoren sprechen ihr auch eine Überlegenheit zur Endoprothese zu [3]. Jedoch ist der Verlust der Gelenkmobilität für viele Patienten nicht zu tolerieren. Deshalb wird von vielen Patienten die Arthrodese auch aus irrationalen Ängsten abgelehnt.

Der heutige Stand der Sprunggelenksendoprothetik erlaubt uns eine Kombination der adäquaten Schmerzreduktion und eine nahezu erhaltene Beweglichkeit des Gelenkes.

Viele Patienten treten an uns heran, denen mehrfach auswärtig eine Arthrodese als Therapieoption angeboten wurde, besonders dann, wenn die benachbarten Gelenke (unteres Sprunggelenk und Chopart'sches) zum Zeitpunkt der Arthrodese nicht gesund sind. Die neuesten Publikationen zeigen gute Resultate nach Endoprothesenimplantation, stammen aber häufig aus den Autorengruppen und verbinden vielfach kleine Fallzahlen und kurze Nachuntersuchungszeiten. 
Waren Sprunggelenksprothesen vor einigen Jahren bei jungen Patienten noch mehr oder weniger kontraindiziert [8], zeigen aktuelle Untersuchungen auch bei ihnen gute Resultate [11]. Die ebenfalls vertretene Meinung, dass zementlose Prothesenmodelle den zementierten in der Haltbarkeit unterlegen sind, wurde widerlegt [9].

Das Prothesendesign hat sich seit der Ersteinführung 1973 verändert. Waren früher viele Prothesen als 2-Komponenten-Modelle konzipiert, zeigte sich in letzter Zeit ein zunehmender Trend zum 3-Komponenten-Modell mit mobilem Inlay (Tab. 1).

\section{Patientengut}

Zwischen 1996 und 2006 wurden in der Orthopädischen Universitätsklinik Magdeburg 570 ESKA-, STAR- und SALTOSprunggelenksendoprothesen implantiert (Abb. 1). Initial begannen wir Erfahrungen mit der ESKA-Prothese (ESKA Implants GmbH \& Co. KG) zu sammeln, arbeiteten dann mit der STAR-Prothese (LINK ${ }^{\circledR}$ S.T.A.R, Waldemar Link, Hamburg, Deutschland) und nahmen dann im Jahr 2001 die SALTO-Sprunggelenksendoprothese (Tornier SA, Saint Ismier, Frankreich) in unser Implantatespektrum auf. Diese beiden letztgenannten Prothesen unterscheiden sich an der Taluskomponente. Hier werden bei der STAR-Prothese alle Seiten künstlich ersetzt, während die SALTO-Prothese medial die natürliche Gelenkfläche beibehält. Seit 1997 implantieren wir in unserer Einrichtung ausschließlich STARund SALTO-Sprunggelenksendoprothesen.

Diese verteilten sich auf 311 Frauen und 259 Männer. Der jüngste Patient war 18 Jahre (mit rheumatoider Arthritis) und der Älteste 84 Jahre (Abb. 2).

Das Durchschnittsalter belief sich auf 55,6 Jahre (18 - 84 Jahre).

In 178 Fällen (31,2\%) bestand zur Aufnahmeuntersuchung eine primäre und in 73 Fällen $(12,8 \%)$ eine Arthrose bei rheumatoider Arthritis. Erwartungsgemäß machen die posttraumatischen Arthrosen mit 319 Fällen (56\%) den Großteil des Patientengutes aus (Abb. 3).

Die mittlere Nachuntersuchungszeit beträgt 4,8 Jahre ( 0,5 - 11 Jahre). Alle unsere Patienten konnten entweder durch

Tab. 1 3-Komponenten-Sprunggelenksprothesen

\begin{tabular}{lll} 
Prothesenmodell & Autor & Jahr \\
\hline LCS & Buechel & 1978 \\
\hline STAR & Kofoed & 1986 \\
\hline Buechel-Pappas & Buechel, Pappas & 1989 \\
\hline SALTO & Judet & 1998 \\
\hline RAMSES & Mendolia & 1998 \\
\hline AES & Asencio & 1998 \\
\hline HINTEGRA & Hintermann & 2000 \\
\hline MOBILITY & Rippstein & 2004
\end{tabular}

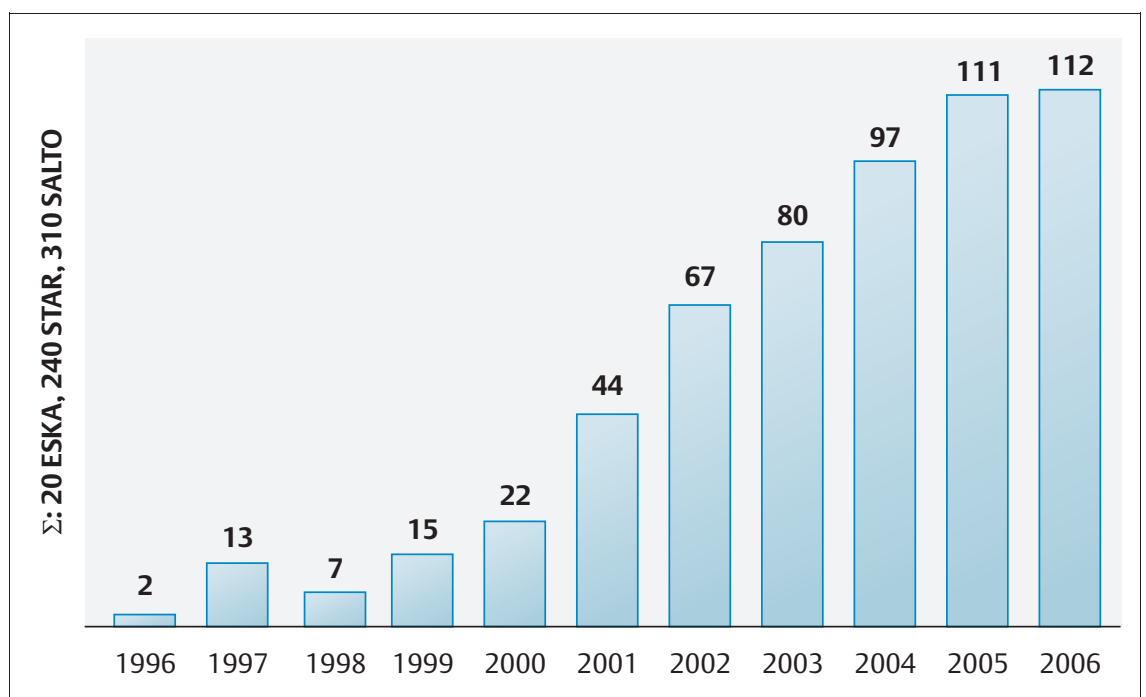

Abb. 1 Implantationshäufigkeit im eigenen Krankengut zwischen 1996 und 2006.

uns, oder bei zu weiten Anreisewegen, durch niedergelassene Kollegen nachuntersucht werden.

\section{Operationstechnik}

Sowohl die STAR-, als auch die SALTOEndoprothese wird über einen ventralen Zugang implantiert. Hierbei ist keine Osteotomie der Fibula (wie bei der ESKA-Prothese) notwendig, was sich unter anderem positiv auf die Nachbehandlung auswirkt.

Die Präparation des Retinaculum extensorum muss subtil durchgeführt werden, da am Ende des Eingriffs eine sichere Naht erreicht werden soll.

Nach Gelenkeröffnung Abtragung eventuell vorhandener ventraler Osteophyten und Platzierung des tibialen Führungsinstrumentariums mit anschließender Pinfixierung. Nach tibialer Re- sektion wird, nach Referenzierung, die entsprechende Resektion am Talusdom vorgenommen. Nach Festlegung und Aufbohrung des Verankerungszapfens erfolgt das Auswählen der Taluskomponente. Nach Schaffung der tibialen Finne Setzen der Probeimplantate und Auswahl des Inlays. Nach einer Bewegungsprüfung Präparation der Verankerung für die tibiale Prothesenkomponente. Bei zufriedenstellender Dorsalextension und Plantarflexion werden dann die Originalimplantate zementlos verankert. Nach Einlage einer RedonDrainage erfolgt der schichtweise Wundverschluss, wobei wir besonderen Wert auf den subtilen Verschluss des Retinaculum extensorum legen. Abb. 4 zeigt das postoperative Bild nach Implantation einer SALTO-Endoprothese. 


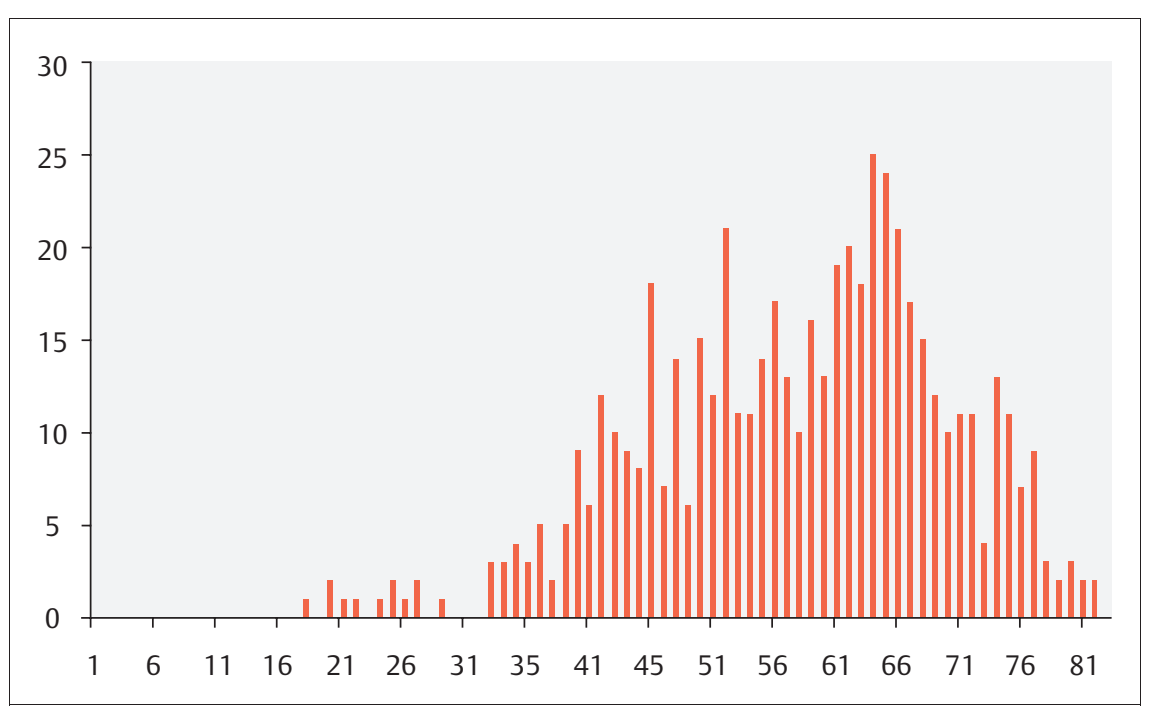

Abb. 2 Altersverteilung

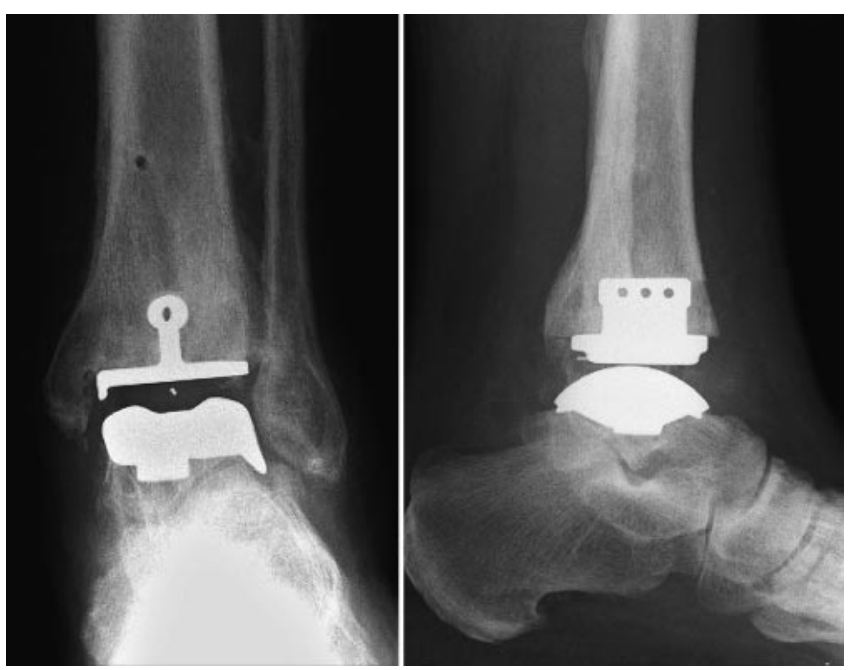

Abb. 4 Postoperatives Bild nach SALTOEndoprothese.

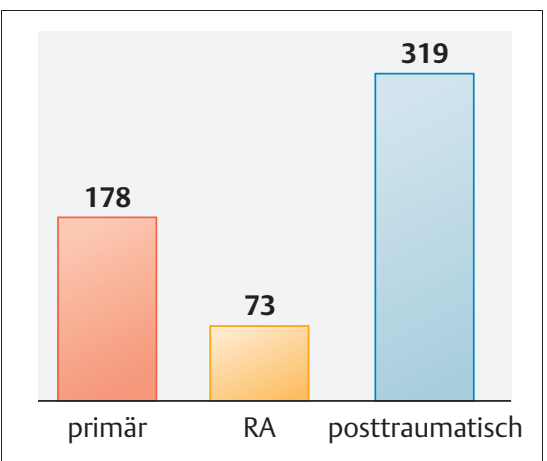

Abb. 3 Äthiologie

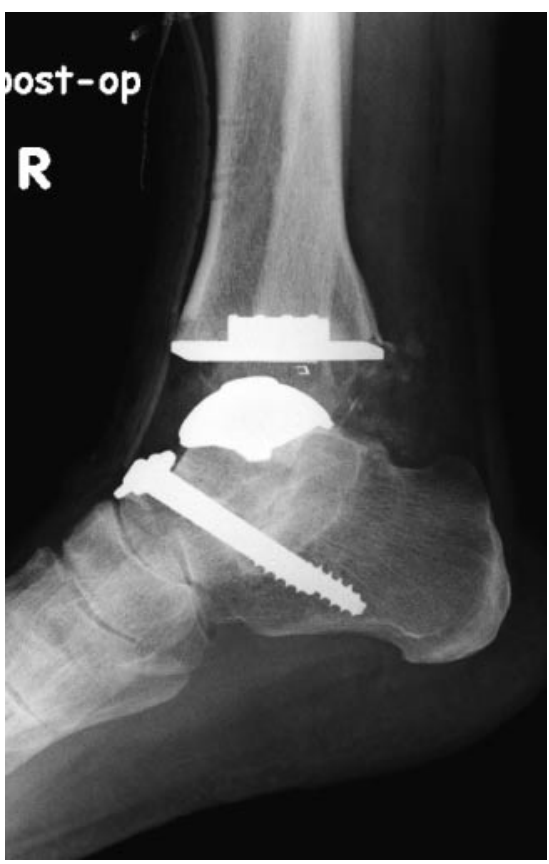

Abb. 5 Postoperative Röntgenkontrolle nach additiver USG-Arthrodese.

eller Lymphdrainage und Kompressionsverbänden. Dieses Vorgehen sehen wir als Hauptursache für die sehr geringe Anzahl an Wundheilungsstörungen (Abb.10). Aufstehen unter Zuhilfenahme von zwei UA-Gehstützen. Bei reizlosen Wundverhältnissen wird nach 8-10 Tagen das Nahtmaterial entfernt und ein zirkulärer Unterschenkelcast oder eine Walker-Orthese angelegt. Hierin ist, bei normalem Heilungsverlauf ohne Komplikationen, eine schmerzadaptierte Vollbelastung erlaubt.

Eine Thromboseprophylaxe wird i.d.R. mit niedermolekularen Heparinen durchgeführt.

Eine ambulante Verlaufskontrolle in unserer Klinik erfolgt 6 Wochen postope- 

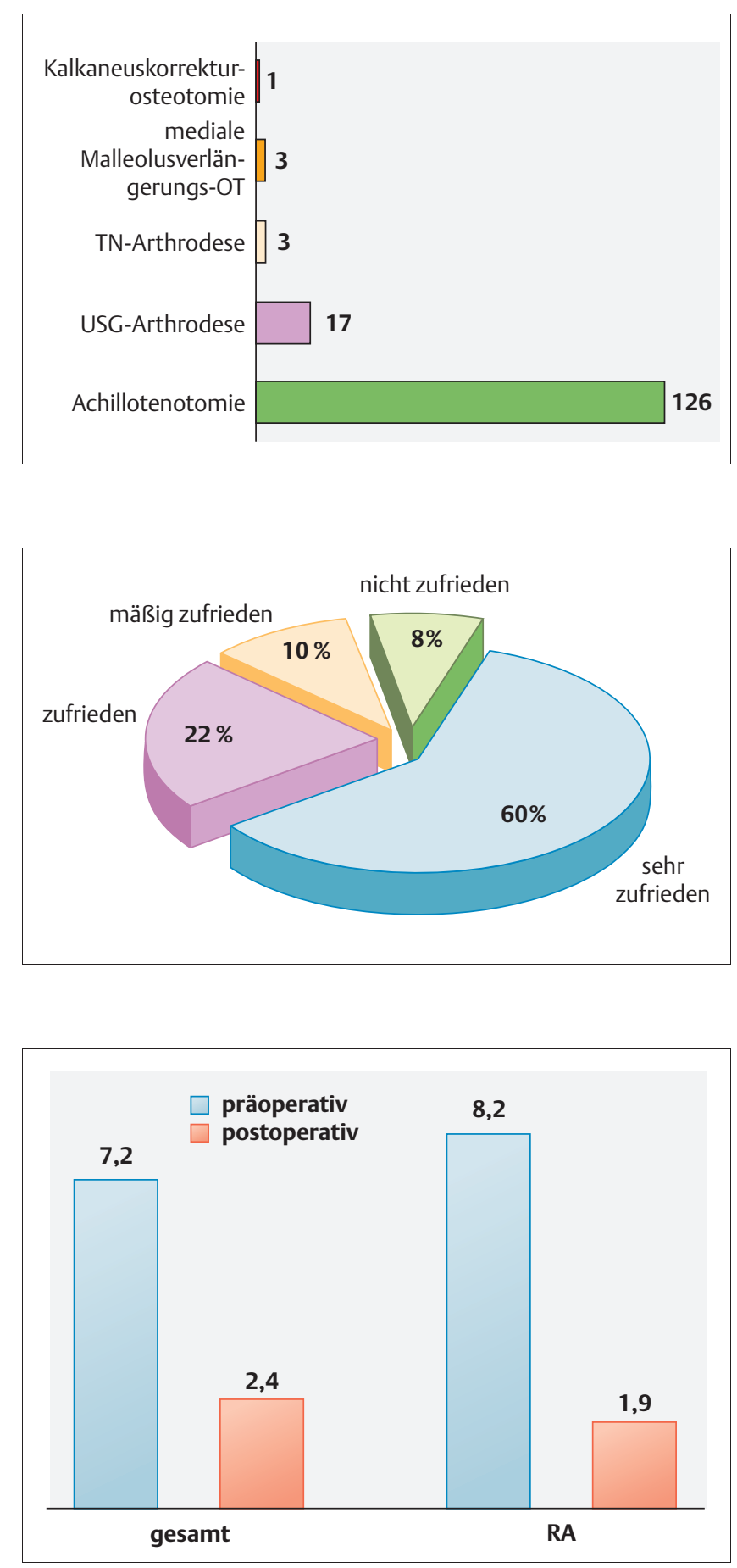

rativ. Nach dieser Zeit wird der Cast bzw. die Walker-Orthese entfernt und eine Röntgenkontrolle durchgeführt. Es folgen eine mehrwöchige Rehabilitationsphase im Rahmen einer stationären $\mathrm{AHB}$ oder eine erweiterte ambulante Physiotherapie.

Eine adäquate und intensive physiotherapeutische Nachbehandlung ist ein entscheidender Faktor für das postoperative Ergebnis.
Abb. 8 Subjektive Schmerzbeurteilung mittels VAS.

Abb. 6 Zusatzeingriffe

Abb. 7 Subjektive Patientenzufriedenheit.

Subjektiv waren $82 \%$ der Patienten mit dem postoperativen Ergebnis zufrieden bzw. sehr zufrieden und nur 18\% mäßig bzw. nicht zufrieden (Abb. 7).

Des Weiteren wurde präoperativ anhand einer visuellen Skala (VAS) das Schmerzniveau der Patienten validiert. Dieses wurde im Rahmen der planmäßigen Nachuntersuchungen wiederholt.

\section{Ergebnisse}

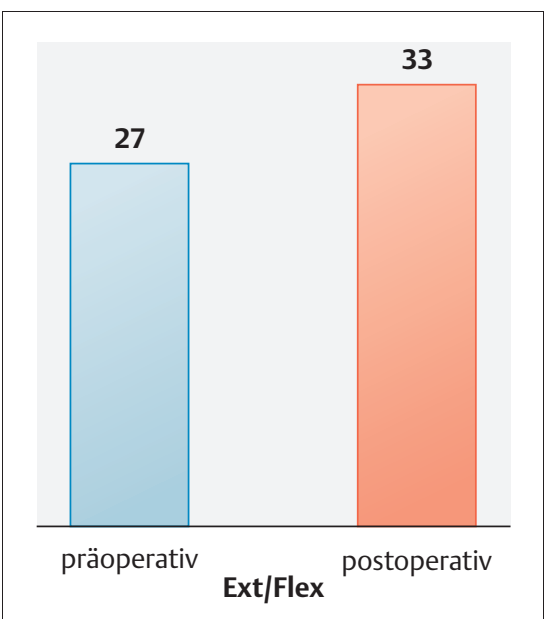

Abb. 9 Prä- und postoperativer ROM (in ${ }^{\circ}$ ).

Es zeigte sich einer Schmerzreduktion von präoperativ 7,2 auf postoperativ 2,4 (Abb. 8). Besonders auffällig sind hier die guten Ergebnisse der Rheumatiker mit einer Reduktion von präoperativ 8,2 auf postoperativ 1,9 (Abb. 8).

Eine Steigerung war ebenfalls im Bereich der Sprunggelenksbeweglichkeit zu beobachten. Es zeigte sich eine Verbesserung des Range of motion (ROM) von präoperativ $27^{\circ}$ auf postoperativ $33^{\circ}$ (Abb. 9), wobei sich eine Korrelation zwischen der präoperativen Beweglichkeit und den postoperativen Ergebnissen zeigte.

\section{Komplikationen}

Bei 37 Patienten (6,5\%) zeigten sich perioperative Komplikationen (Abb.10).

In 23 Fällen (4\%) kam es intraoperativ zu einer medialen Malleolusfraktur, welche wir sowohl konservativ im Gips als auch mit einer primären Osteosynthese (Schrauben, K-Drähte) behandelten. Bei diesen Patienten wurde das Nachbehandlungsprogramm nur in der postoperativen Belastung (Teilbelastung) modifiziert. Alle Frakturen heilten ohne Pseudarthrose aus. Des Weiteren zeigten 12 Patienten (2,1\%) eine postoperative Wundheilungsstörung. Bei 2 Patienten $(0,4 \%)$ wurde postoperativ eine Läsion des N. fibularis (Ramus superficialis) festgestellt. Diese war nur partiell und bildete sich im Verlauf vollständig zurück.

71 Patienten (12,5\%) mussten sich aufgrund von postoperativen Komplikationen oder persistierenden Schmerzen 


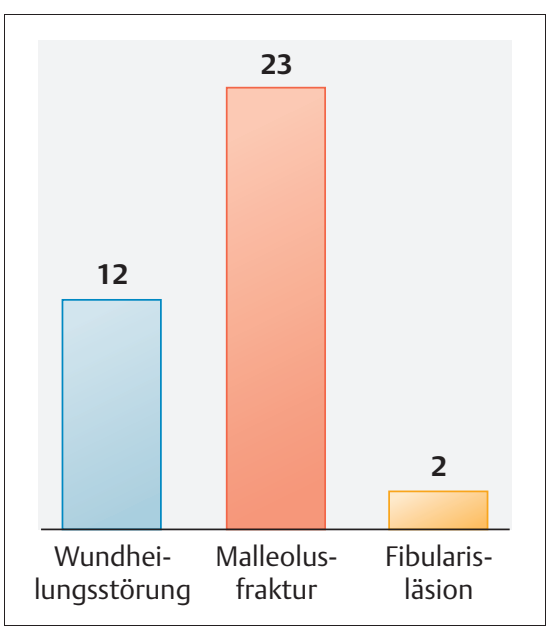

Abb. 10 Komplikationen

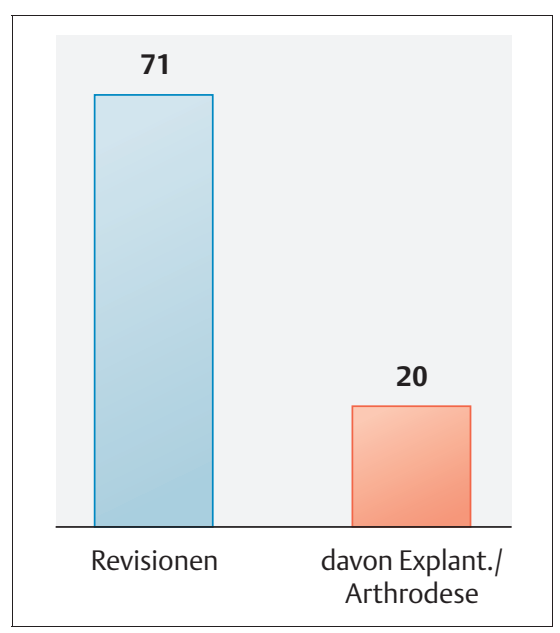

Abb. 11 Revisionen
Tab. 2 Literaturübersicht

\begin{tabular}{lcrllc} 
Autor & Jahr & $\mathbf{n}$ & $\begin{array}{l}\text { NU } \\
\text { (Jahre) }\end{array}$ & $\begin{array}{l}\text { Ergebnisse } \\
\text { (\%) } \\
\text { (sehr gut) }\end{array}$ & $\begin{array}{l}\text { Revisionen } \\
\text { (\%) }\end{array}$ \\
\hline Pyevich & 1998 & 98 & 4,8 & 83 & 5,1 \\
\hline Schill & 2002 & 33 & 4,1 & 94 & 6 \\
\hline Wood & 2003 & 200 & 3,8 & 73 & 11 \\
\hline Anderson & 2003 & 51 & $3-8$ & 61 & 23,5 \\
\hline Hinterman & 2004 & 122 & $1-3$ & 84 & 6,6 \\
\hline Kofoed & 2004 & 58 & 9,4 & & 17,2 \\
\hline Hinterman & 2006 & 278 & 8,3 & & 14,4 \\
\hline Ali & 2007 & 35 & 5 & 97 & 2,9
\end{tabular}

einer Revision unterziehen (Abb.11). Ursache für eine Revision konnte unter anderem eine Impingementsymptomatik, eine Lockerung der tibialen bzw. talaren Prothesenkomponente, Talusnekrosen oder tiefe Infektionen sein. Anteilig waren die Revisionen infolge einer Impingementsymptomatik (besonders bei posttraumtischen Arthrosen) am häufigsten vertreten. Patienten mit einer rheumatoiden Arthritis mussten in keinem Fall wegen eines Impingement revidiert werden.

Von diesen 71 Patienten erfolgte in 20 Fällen (3,5\%) eine Konversion auf eine Arthrodese (Abb.11).

\section{Diskussion}

Während früher mit zunehmenden Standzeiten der Endoprothesen die Lockerungsraten stiegen [16], zeigen sich in den letzen Jahren bessere Mittel- und
Langzeitergebnisse nach Implantation $[1,2,5,6,10,14,15,17]$. Eine Literaturübersicht zeigt Tab. 2. Unsere eigenen Erfahrungen bestätigen diese Ergebnisse.

Sicherlich haben sowohl die Fortschritte im Prothesendesign, als auch die stark verbesserten Instrumentarien einen großen Anteil an diesem Erfolg. Optimierte Oberflächenbeschichtungen der Komponenten zeigen ein besseres Osteointegrationsverhalten [16] und der Übergang zu einer zementlosen Fixation gestattet eine sparsamere Resektion mit einem geringeren lokalen Trauma durch Hitzenekrosen. Trotzdem sind noch viele Fragen in puncto Prothesendesign offen und zu klären.

Die in der Literatur oftmals beschriebene Problematik der Wundheilungsstörung konnten wir im eigenen Krankengut kaum beobachten.
Unsere geringe Komplikationsrate führen wir auf die sorgfältige und vollständige Rekonstruktion des Retinakulums bei gleichzeitiger Gelenkdrainage, unser postoperatives Wundmanagement (Ruhigstellung in Walker-Orthese/Gips), sowie unsere gute Zusammenarbeit mit der physiotherapeutischen Abteilung (inkl. Lymphdrainage) zurück.

Ein weiterer wichtiger Punkt ist die „learning curve“. Während wir bei den ersten 50 implantierten Prothesen eine Frühkomplikations-/Revisionsrate von $22 \%$ hatten, reduzierte sie sich in den Folgejahren auf 6\%. Auch Henricson et al. beschrieben in den ersten 4 Jahren Revisionsraten von 33\%, welche sich mit größeren Implantationszahlen auf $15 \%$ reduzierten [4].

Ebenfalls muss man sich vor Augen führen, dass das postoperative Outcome stark von der Arthroseäthiologie abhängig ist. Die Ergebnisse bei Patienten mit rheumatoider Arthritis sind sowohl in puncto Schmerzreduktion als auch in der Beweglichkeit gut. Im Gegensatz dazu zeigen Patienten mit einer posttraumatischen Arthrose eine im Vergleich dazu schlechtere postoperative Beweglichkeit.

Des Weiteren zeigen unsere Untersuchungen, dass das Outcome nach Implantation einer Sprunggelenksendoprothese bei primärer Arthrose abhängig von der präoperativen tibiotalaren Achse ist. So zeigte sich, das die Implantation einer Endoprothese bei Fehlstellungen (varus/valgus) von mehr als $15^{\circ}$ zu einer Revisionsrate von über 33\% führte. In diesen Fällen empfehlen wir eine supramalleoläre Korrekturosteotomie der Tibia, der im späteren Verlauf eine Sprunggelenkendoprothese folgen kann. In den letzten 5 Jahren führten wir dieses Prozedere in 3 Fällen von insgesamt 42 supramalleolären Korrekturosteotomien durch.

Die Sprunggelenksendoprothetik sollte kein Eingriff sein, der wenige Male im Jahr durchgeführt wird. Kontinuierliche Implantation sichert ein adäquates $\mathrm{Er}$ fahrungslevel!

Dies umso mehr, als die OP-Technik und die Weichteilbehandlung wichtiger $\mathrm{zu}$ sein scheinen, als die in den letzten Jahren sprunghaft wachsende Zahl verschiedenster Endoprothesenmodelle. 


\section{Literatur}

1 Ali MS, Higgins GA, Mohamed M. Intermediate results of Buechel Pappas unconstrained uncemented total ankle replacement for osteoarthritis. J Foot Ankle Surg 2007; 46: 16 20

2 Anderson T, Montgomery F, Carlsson A. Uncemented STAR total ankle prostheses. Three to eight-year follow-up of fifty-one consecutive ankles. J Bone Joint Surg [Am] 2003; 85: $1321-1329$

${ }^{3}$ Bolton-Maggs BG, Sudlow RA, Freeman MA Total ankle arthroplasty. A long-term review of the London Hospital experience. J Bone Joint Surg [Br] 1985; 67: 785 - 790

${ }^{4}$ Henricson A, Agren P-H. Secondary surgery after total ankle replacement. The influence of preoperative hindfoot alignement. J Foot Ankle Surg 2007; 13: $41-44$

${ }^{5}$ Hintermann B, Valderrabano V, Dereymaeker G, Dick W. The HINTEGRA ankle: rationale and short-term results of 122 consecutive ankles. Clin Orthop Relat Res 2004; 424: 57-68

${ }^{6}$ Hintermann B, Valderrabano V, Knupp M, Horisberger M. [The HINTEGRA ankle: shortand mid-term results]. Orthopäde 2006; 35 : 533-545

7 Jerosch J, Fayaz H, Senyurt H. [Ankle arthrodesis versus ankle replacement: a comparison]. Orthopade 2006; 35: 495-505
${ }^{8}$ Kitaoka HB, Patzer GL, Ilstrup DM, Wallrichs SL. Survivorship analysis of the Mayo total ankle arthroplasty. J Bone Joint Surg [Am] 1994; 76: $974-979$

${ }^{9}$ Kofoed H. [The evolution of ankle arthroplasty]. Orthopade 1999; 28: 804-811

10 Kofoed H. Scandinavian Total Ankle Replacement (STAR). Clin Orthop Relat Res 2004; $73-79$

${ }^{11}$ Kofoed H, Lundberg-Jensen A. Ankle arthroplasty in patients younger and older than 50 years: a prospective series with long-term follow-up. Foot Ankle Int 1999; 20: 501 -506

12 Lord G, Marotte JH. [Total ankle prosthesis. Technic and 1st results. Apropos of 12 cases]. Rev Chir Orthop Reparatrice Appar Mot 1973; 59: 139-151

${ }^{13}$ McGuire MR, Kyle RF, Gustilo RB, Premer RF. Comparative analysis of ankle arthroplasty versus ankle arthrodesis. Clin Orthop Relat Res 1988; 226: 174- 181

14 Pyevich MT, Saltzman CL, Callaghan JJ, Alvine FG. Total ankle arthroplasty: a unique design. Two to twelve-year follow-up. J Bone Joint Surg [Am] 1998; 80: 1410-1420

${ }^{15}$ Schill S, Biehl C, Thabe H. [Ankle prostheses. Mid-term results after Thompson-Richards and STAR prostheses]. Orthopäde 1998; 27: $183-187$

16 Thermann H, Saltzman CL. [Prosthetic replacement of the upper ankle joint]. Unfallchirurg 2002; 105: 496-510

17 Wood PL, Deakin S. Total ankle replacement. The results in 200 ankles. J Bone Joint Surg [Br] 2003; 85: 334-341

\section{Dr. med. Sebastian Lieske}

Assistenzarzt

\section{Dr. med. Katja Schenk}

Oberärztin

Dr. med. Michael John

Facharzt/Stationsarzt

Prof. Dr. med. Hans Wolfram Neumann

Ärztlicher Direktor

Orthopädische Universitätsklinik

Otto-von-Guericke-Universität

Magdeburg

Universitätsklinikum Magdeburg A. ö.R.

Leipziger Straße 44

39120 Magdeburg 